3. Kallen M, Hornick J. The 2020 WHO Classification; what's new in soft tissue pathology? Am J Surg Pathol 2020; 45: e1-23.

\section{BRAF MUTATIONS IN LOW-GRADE GLIOMAS; A CASE REPORT}

Thomas de Hoog, Karen Whale

Department of Anatomical Pathology, Royal Hobart Hospital, Hobart, Tas, Australia

$B R A F$ mutations play an important role in the classification and prognostication of low-grade gliomas and may offer a therapeutic target. We report a case of a V600E mutant low-grade glioma with morphological features of a ganglioglioma diagnosed in a 22-year-old female. Sections demonstrated a lowgrade lesion with atypical astrocytic glial and neuronal elements, the latter stained positive for chromogranin A and BRAF V600E immunohistochemical stains; IDH-1 immunohistochemistry was negative. A provisional diagnosis of ganglioglioma (WHO grade I) was made with a differential diagnosis of pilocytic astrocytoma with gangliocytic differentiation. Subsequent molecular analysis confirmed the tumour to be $I D H$ wild-type and contain a BRAF V600E point mutation, with absence of KIAA1549-BRAF fusion, supporting the initial diagnosis of a ganglioglioma. $B R A F$ $V 600 E$ point mutations are well recognised as a major genetic alteration in low grade paediatric glial tumours and confer prognostic information. Additionally, there is emerging evidence that BRAF and MEK inhibitors may have a therapeutic role in $B R A F$ mutant gliomas.

\section{References}

1. Behling F, Schittenhelm J. Oncogenic BRAF alterations and their role in brain tumours. Cancers (Basel) 2019; 11: 794.

2. Nobre L, Zapotocky M, Ramaswamy V, et al. Outcomes of BRAF V600E pediatric gliomas treated with targeted BRAF inhibition. JCO Precis Oncol 2020; 4: 561-71.

\section{SMARCA4-DEFICIENT NON-SMALL CELL LUNG CANCER}

Alyona Dziouba ${ }^{1}$, Andrew Dettrick $^{1}$, Anthony Gill ${ }^{2}$ ${ }^{1}$ Pathology Queensland, Sunshine Coast University Hospital, Qld, Australia; ${ }^{2}$ NSW Health Pathology, Royal North Shore Hospital, NSW, Australia

Background: SMARCA4-deficient malignancies are high grade, poorly differentiated tumours with aggressive behaviour and poor prognosis. ${ }^{1}$

Case report: We report the case of a 41-year-old male with SMARCA4 deficient metastatic non-small cell lung cancer (NSCLC) diagnosed on brain resection. We reviewed the imaging, clinical records, and histopathology available on the electronic medical record. The patient presented with a right sided hemi-anaesthesia. On brain imaging, a $30 \mathrm{~mm}$ mass in the left thalamus and peduncle was found and the patient proceeded to resection.

Histopathology: The histopathology showed a metastatic poorly differentiated carcinoma. Despite extensive intrathoracic lymph node involvement on staging imaging, no definitive lung primary was identified. Initial immunohistochemistry (IHC) for tumour characterisation revealed a profile compatible with a lung primary, favour NSCLC. Further IHC to delineate mutation status revealed a SMARCA4 deficiency.

Outcome: Despite extensive treatment the patient progressed rapidly and died in a palliative care unit, 7 months post diagnosis. Discussion: Current evidence suggests that the great majority of SMARCA4-deficient malignancies arising in the thorax represent smoking related undifferentiated/de-differentiated carcinomas rather than primary thoracic sarcomas. ${ }^{2}$ Patients with SMARCA4 deficiency have worse survival rates regardless of stage of disease, thus, awareness of this entity is becoming increasingly important. ${ }^{1}$

Acknowledgement: Dr Krishnan Iyengar, Pathology Queensland, Royal Brisbane and Women's Hospital, Queensland, Australia.

\section{References}

1. Amron S, Hofman P, Ilie M. Perspectives and issues in the assessment of SMARCA4 deficiency in the management of lung cancer patients. Cells 2021; 10: 1920.

2. Rekhtman N, Montecalvo J, Chang JC, et al. SMARCA4deficient thoracic sarcomatoid tumours represent primarily smoking-related undifferentiated carcinomas rather than primary thoracic sarcomas. J Thorac Oncol 2020; 15: 231-47.

\section{SEVERE TERMINAL ILEITIS FOLLOWING SARS-COV-2 INFECTION: A HISTOPATHOLOGICAL CASE REPORT}

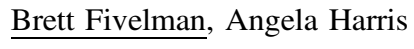

Anatomical Pathology (SEALS), NSW Health Pathology East, Prince of Wales Hospital, Sydney, NSW, Australia

In children COVID 19 usually produces mild respiratory symptoms. However, in a rare subset of paediatric patients a severe post-infectious hyperinflammatory syndrome may arise, termed multisystem inflammatory syndrome in children (MISC). Gastrointestinal manifestations are particularly common and may mimic other gastrointestinal infections or inflammatory bowel disease. ${ }^{1}$ There is a paucity of literature describing the histological features of intestinal inflammation in MIS-C. ${ }^{2}$ We report the case of a 15-year-old male who presented with right iliac fossa pain and raised serum inflammatory markers in the context of recent acute encephalopathy following SARS-CoV-2 infection. The patient underwent ileocolic resection. Histological analysis demonstrated chronic active enteritis with granulomatous inflammation, stricture formation, fissures and ileal perforation. Long term studies are needed to understand the risk of future bowel disease in patients with gastrointestinal manifestations of MIS-C.

\section{References}

1. Gómez IJA, López PP, Duque DC, et al. Abdominal manifestation of multisystemic inflammatory syndrome in children. J Pediatr Surg Case Rep 2021; 74: 102042.

2. Sahn B, Eze OP, Edelman MC, et al. Features of intestinal disease associated with COVID-related multisystem inflammatory syndrome in children. $J$ Pediatr Gastroenterol Nutr 2021; 72: 384 .

\section{MASSIVE RETINAL GLIOSIS MIMICKING AN INTRAOCULAR NEOPLASM}

Charlotte Foster ${ }^{1}$, Tim Sutton $^{1}$, Amanda Charlton ${ }^{2}$ 